Gefässchirurgie 2016 $21: 411-417$ DOI 10.1007/s00772-016-0189-8

Online publiziert: 30 . September 2016 (c) Der/die Autor(en) 2016. Dieser Artikel ist eine Open-Access-Publikation.

CrossMark

\author{
D. Baumgartner ${ }^{1} \cdot$ Y. von Kodolitsch ${ }^{2}$ \\ ' Univ.-Klinik für Kinder- und Jugendheilkunde, Klinische Abteilung für Pädiatrische Kardiologie, \\ Medizinische Universität Graz, Graz, Österreich \\ ${ }^{2}$ Universitäres Herzzentrum Hamburg, Klinik und Poliklinik für Allgemeine und Interventionelle \\ Kardiologie, Universitätsklinikum Hamburg-Epppendorf, Hamburg, Deutschland
}

\title{
Aortale Bildgebung als Schlüssel zur Therapie genetischer Aortenerkrankungen
}

\section{Einleitung}

Die bildgebende Diagnostik spielt in der Betreuung von Patienten mit genetischen Aortenerkrankungen eine essenzielle Rolle. Zwei klinische Szenarien können vorliegen:

1. Äußerliche Zeichen einer Bindegewebeschwäche im Skelettsystem, an den Augen, an der Haut oder ein Pneumothorax lassen eine Aortopathie vermuten. Eine Aortendilatation meist im Bereich der Aortenwurzel ist $\mathrm{zu}$ beweisen oder auszuschließen. Ihre Form - es dominiert entweder die Dilatation des Bulbus aortae oder der Aorta ascendens - macht unterschiedliche Ursachen wahrscheinlich.

2. Schmerzen im Thorax oder Schocksymptomatik weisen auf eine lebensbedrohliche Aortendissektion mit der Gefahr einer Aortenruptur hin. Eine rasche, detaillierte Diagnostik ist die Voraussetzung für die Planung der Akutbehandlung, sei sie eine Notfalloperation mit Aortenwurzelersatz oder eine konservative Stabilisierung bei Typ-B-Dissektion.

Aortenaneurysma und Aortendissektion zählen mit Abstand zu den lebensgefährlichsten Manifestationen des MarfanSyndroms (MFS). Aortopathien treten beim Marfan-Syndrom vor allem in der proximalen Aorta auf, können aber prinzipiell in jedem Abschnitt der Aorta auftreten und entwickeln sich altersabhängig. Deshalb ist ein lebenslanges Monitoring mittels Echokardiographie oder einem anderen bildgebenden Verfahren notwendig. Die Dilatation der Sinus valsalvae kann bereits in utero beginnen, bei anderen Patienten erreicht die Aortendilatation nie ein Ausmaß, das eine chirurgische Therapie erforderlich macht. Meistens ist die Aortenwurzel, also der Bereich der Sinus valsalvae, am stärksten betroffen, wobei eine zwiebelförmige Dilatation charakteristisch ist. Oft ist zudem auch die proximale Aorta aszendens mit betroffen, indem es $\mathrm{zu}$ einem Verstreichen des sinutubulären Übergangs kommt. Dissektionen der Aorta können jedoch an unterschiedlichen Stellen der Aorta deszendens auftreten. Die Interpretation von Aortendurchmessern erfordert den Vergleich mit altersspezifischen Nomogrammen und das in Beziehung setzen des Aortendurchmessers mit der Körperoberfläche oder der Körpergröße [1].

\section{Klinische Zeichen der Aortopathie}

Klinisch zeigt sich eine Aortendissektion meistens durch anhaltende Thoraxschmerzen mit Ausstrahlung entlang des Dissektionsverlaufes [2]. Sie beginnt meist im Bereich oberhalb des Sinus valsalvae (Stanford Typ A) und bleibt entweder auf die Aorta aszendens beschränkt (Debakey Typ II), oder breitet sich weiter in die deszendierende Aorta aus (Stanford Type A; Debakey Typ I) [3].

Eine akute kardiale Dekompensation weist in diesem Zusammenhang ent- weder auf eine Perikardtamponade hin oder besteht aufgrund einer Aortenklappeninsuffizienz, die durch den Prolaps einer Tasche der Aortenklappe durch das Dissekat entstehen kann. Sind die Karotiden mit vom Dissekat betroffen, kann es zu neurologischen Symptomen kommen, sind die Koronararterien involviert, können Myokardinfarkte oder plötzlicher Herztod die Folge sein. Wenn das Aneurysma in das Perikard rupturiert, entsteht eine Herzbeuteltamponade. Chronische Aortendissektionen verlaufen oft schleichend und tückischerweise ohne Thoraxschmerz.

\section{Kriterien für den elektiven Ersatz der Aorta}

Ein chirurgischer Aortenwurzelersatz wird bei Erwachsenen mit Marfan-Syndrom meist bei einem Aortendurchmesser von $50 \mathrm{~mm}$ empfohlen $[4,5]$. Liegt in der Familienanamnese eine Aortendissektion in jungem Alter vor, ist eine frühere Indikationsstellung ratsam. Für das Stellen der Operationsindikation im Kindesalter existieren keine eindeutigen Leitlinien. Eine rasche Aortendurchmesserzunahme ( $>0,5 \mathrm{~cm}$ pro Jahr) und Aortenklappeninsuffizienz sind Kriterien für ein früheres operatives Eingreifen. Der Umstand, dass Aortendissektionen und Aortenrupturen in einer Studie an 675 Patienten schon bei Aortendurchmessern unter $30 \mathrm{~mm}$ beschrieben wurden [5], macht klar, dass neben den Aortendurchmessern weitere, die Aortenwandschädigung charakterisierende 
Tab. 1 Überblick über die empfohlene Bildgebung bei verschiedenen erblichen Aortopathien

\begin{tabular}{|c|c|c|c|c|}
\hline \multirow{2}{*}{$\begin{array}{l}\text { Empfehlungen } \\
\text { zur Basisbildge- } \\
\text { bung }\end{array}$} & \multirow{2}{*}{$\begin{array}{l}\text { TTE } \\
\text { Aortenwurzel }\end{array}$} & \multicolumn{3}{|l|}{ MRA oder CTA } \\
\hline & & $\begin{array}{l}\text { Aorta } \\
\text { thorako-abdo- } \\
\text { minell }\end{array}$ & $\begin{array}{l}\text { Arterien tho- } \\
\text { rakal, viszeral, } \\
\text { Becken }\end{array}$ & $\begin{array}{l}\text { Arterien zervi- } \\
\text { kal, und zere- } \\
\text { bral }\end{array}$ \\
\hline BAV & Empfohlen & Empfohlen & Nicht empfohlen & $\begin{array}{l}\text { Nicht } \\
\text { empfohlen }\end{array}$ \\
\hline MFS & Empfohlen & Empfohlen $^{\mathrm{b}}$ & Nicht empfohlen & Nicht empfohlen \\
\hline LDS & Empfohlen & Empfohlen $^{\mathrm{b}}$ & Empfohlen & Empfohlen \\
\hline vEDS & Empfohlen & Empfohlen & Empfohlen & Empfohlen \\
\hline TS & Empfohlen $^{d}$ & Empfohlen $^{d}$ & Nicht empfohlen & Nicht empfohlen \\
\hline
\end{tabular}

BAV bikuspide Aortenklappenenerkrankung, CoA Aortenisthmusstenose, CTA computertomographische Angiographie, LDS Loeys-Dietz-Syndrom, MFS Marfan-Syndrom, MRA Magnetresonanzangiographie, TS Turner-Syndrom, TTE transthorakale Echokardiographie, vEDS vaskuläres Ehlers-Danlos-Syndrom

${ }^{a}$ Risiko für Hirnaneurysmen ist erhöht, Empfehlungen zum Imaging gibt es jedoch nicht

'Inklusive Vermessung der proximalen Pulmonalarterie

'Eine Invasive Diagnostik arterieller Gefäße sollte aufgrund häufiger Komplikationen am Punktionsort und im Zielgefäß möglichst vermieden werden

Inklusive Untersuchung auf FehImündung der Pulmonalvenen

Parameter für die Operationsindikation von Nutzen sind.

\section{Bildgebung}

Für die Bildgebung der Aorta stehen die in - Tab. 1 aufgelisteten Methoden zur Verfügung.

\section{Die transthorakale Echokardiogra- phie}

Der Vorteil dieser Methode liegt in der breiten Verfügbarkeit und Wiederholbarkeit aufgrund fehlender Invasivität und Strahlenbelastung. Diese Methode eignet sich für Screening-Untersuchungen, d. h. der Abklärung von Verdachtsfällen, für Verlaufsuntersuchungen von Patienten jeder Altersgruppe und für funktionelle Untersuchungen der Aortenwandelastizität. Das zweidimensionale Bild liefert mit Transducerposition links hoch parasternal und suprasternal einen Gesamteindruck des Bulbus aortae und des Aortenbogens in Relation zu den umgebenden Strukturen wie Aortenklappe, AV-Klappen, Ventrikel und Vorhöfe. Die Farbdopplersonographie lässt schon im Kindesalter oft eine zentrale Insuffizienz der Aortenklappe erkennen, die durch eine Dilatation des Aortenklappenrings bedingt ist. Die M-Mode-Echokardiographie zeigt mit einer hohen örtlichen und zeitlichen Auflösung den Aorten- durchmesser im Verlauf des Herzzyklus auf verschiedenen definierten Höhen der Aorta. Aufgrund der Aortenwandelastizität ist die zeitliche Einordnung der gemessenen Aortendurchmesser im Herzzyklus für die Vergleichbarkeit der Messwerte von großer Bedeutung. Dazu dient das ins Ultraschallsystem integrierte EKG mit seinem QRS-Komplex als Orientierung für die Enddiastole. Nicht zuverlässig dargestellt werden kann in der transthorakalen Echokardiographie der hinter der Lunge verlaufende Abschnitt der thorakalen Aorta.

\section{Die transösophageale Echokardiographie}

Die transösophageale Echokardiographie überwindet diese Limitationen der transthorakalen Ausschallung, bei der die Abbildung der Aorta von der Aortenwurzel bis knapp unter das Zwerchfell möglich ist. Sie ist der transthorakalen Echokardiographie vor allem wegen des größeren Schallfensters überlegen, das nicht durch Rippen unterbrochen ist. Durch die Nachbarschaft zum Bronchialsystem ist die distale Aorta ascendens allerdings ein „blinder Fleck“ der TEE. Eine Stärke der Methode besteht insbesondere darin, dass sie schnell verfügbar ist, Sie ist wenig invasiv und bietet sich an, um im Notfall eine Aortendissektion vor allem bei hämodynamisch instabilen Patienten rasch nachzuweisen.

\section{Das CT mit CT-Angiographie}

Das CT mit CT-Angiographie ist eine exakte und schnelle Methode, im Notfall eine Aortendissektion zuverlässig zu diagnostizieren oder auszuschließen. Für die präoperative Planung liefert das $\mathrm{CT}$ zusätzlich Informationen über die Koronargefäße. Auch zur früh postoperativen Erfolgsbeurteilung der Chirurgie hat das CT seine Berechtigung. Die damit verbundene Strahlenbelastung limitiert den verantwortungsbewussten Einsatz des CTs insbesondere bei Kindern, Schwangeren und jungen Patienten, die viele serielle Untersuchungen im Laufe ihres Lebens benötigen. Um eine optimale örtliche Auflösung der Strukturen zu erreichen, ist bei Kindern vor dem 6. Lebensjahr für Schnittbilduntersuchungen in der Regel eine Sedierung oder Narkose notwendig.

Die Magnetresonanztomographie (MRI) mit MR-Angiographie (MRA)

Die Magnetresonanztomographie (MRI) mit MR-Angiographie (MRA) konnte in vielen Bereichen das $\mathrm{CT}$ ablösen und so das Risiko für durch ionisierende Röntgenstrahlung induzierte maligne Erkrankungen deutlich senken. CT und MRI haben einen gemeinsamen großen Vorteil gegenüber der Herzultraschalluntersuchung. Beide Methoden ermöglichen die Darstellung der gesamten Aorta von der Aortenklappe bis zur Iliakalbifurkation. Vor jeder Operation der thorakalen Aorta sollte jegliche Pathologie der distal verbleibenden Restaorta bekannt sein, um das Risiko für postoperative Komplikationen und Folgeeingriffe besser einschätzen zu können.

Die Aortenquerschnittsbilder zeigen den Beginn und das Ende der Dissektion, den Verlauf der Dissektionsmembran und alle Kommunikationen zwischen wahrem und falschem Aortenlumen sowie, von welchem der beiden Aortenlumina die essenziellen Aortenäste Truncus coeliacus, Arteria mesenterica superior und die Nierenarterien entspringen. Diese Informationen sind mit 
klinischen Symptomen wie Sehstörungen, Angina abdominalis und Zeichen der Nierenminderperfusion in Einklang zu bringen. Eine 3-D-Rekonstruktion der gesamten Aorta liefert einen Gesamtüberblick über die Aorta, die elongiert und geschlängelt sein kann oder aufgrund einer begleitenden Skoliose bogig verläuft.

Durch die zunehmende Lebenserwartung und Lebensqualität bei genetischen Aortenerkrankungen treten mehr und mehr Patientinnen mit Aortopathie mit dem Wunsch an den betreuenden Arzt heran, in einer geplanten Schwangerschaft kardiologisch betreut zu werden. Basis für die Beratung und gemeinsame informierte Entscheidungsfindung sollte stets eine umfassende Bildgebung der gesamten Aorta sein. Das MRI bietet sich als Basisbildgebung vor einer Schwangerschaft an. Obwohl keine Studien vorliegen, dass das verwendete starke Magnetfeld von 1,5 oder $3 \mathrm{~T}$ embryonale Schäden hervorruft, sollte das erste Trimenon wenn möglich von einer MR-Untersuchung ausgespart bleiben. Ab dem 2. Trimenon ist eine MR- Untersuchung der Aorta gut vertretbar, insbesondere dann, wenn vor der Schwangerschaft kein anatomisches Mapping der Aorta erfolgt ist, oder wenn eine solche Bildgebung aufgrund anatomischer Charakteristika der Aortenerkrankung notwendig ist. Während der Schwangerschaft sollte allerdings auf die Gabe von Gadolineum als Kontrastmittel verzichtet werden, da nicht klar ist, ob die Substanz in das fetale Gewebe eingebaut wird.

\section{》) Das erste Trimenon sollte von einer MR-Untersuchung ausgespart bleiben}

Generell gibt es die Empfehlung, eine reine Aortenektasie mittels MRI ohne Kontrastmittel, eine Dissektion oder postoperative Situation nach Aortenwurzelersatz mit Kontrastmittel darzustellen, um wahre und falsche Lumina gegebenenfalls gut differenzieren zu können (• Abb. 1).

Für die Beurteilung von Messungen der Aortendurchmesser oder Querschnittsflächen muss jede Messung auf

Gefässchirurgie 2016 21:411-417 DOI 10.1007/s00772-016-0189-8

(c) Der/die Autor(en) 2016. Dieser Artikel ist eine Open-Access-Publikation.

D. Baumgartner $\cdot$ Y. von Kodolitsch

\section{Aortale Bildgebung als Schlüssel zur Therapie genetischer Aortenerkrankungen}

\section{Zusammenfassung}

Hintergrund. Die bildgebende Diagnostik spielt in der Betreuung von Patienten mit genetischen Aortenerkrankungen wie dem Marfan-Syndrom, dem vaskulären Ehlers-Danlos-Syndrom oder dem LoeysDietz-Syndrom sowohl bei Diagnose der genetischen Aortenerkrankung im stabilen Stadium als auch bei der Notfalldiagnostik akuter Aortensyndrome eine essenzielle Rolle. Die bildgebenden Modalitäten zur elektiven Diagnostik und Akutdiagnostik umfassen die transthorale Echokardiographe, die transösophageale Echokardiographie, die CT mit CT-Angiographie und die Magnetresonanztomographie (MRI) mit MRAngiographie (MRA).

Fragestellung. Stellenwert der M-Modeechokardiographischen Bildsegmentationsmethode zur Messung von Steifigkeitsveränderungen der Aorta.
Material und Methoden. Studie zur Steifheitsmessung der Aorta bei Marfan-Syndrom im Vergleich zu gesunden Probanden. Ergebnisse

Mit der nichtinvasiven, semiautomatisierten M-Mode-echokardiographischen Bildsegmentationsmethode kann die Verminderung der elastischen Eigenschaften der Aorta bei jungen Patienten mit Marfan-Syndrom genau und objektiv erfasst werden.

Schlussfolgerungen. Bildgebende Verfahren sind zur Risikostratifikation und zur Akutdiagnostik genetischer Aortensyndrome unverzichtbar. Aus Strahlenschutzgründen sollen für Verlaufsuntersuchungen junger Patienten bevorzugt Echokardiographie und MRT eingesetzt werden.

Schlüsselwörter

Marfan-Syndrom · Echokardiographie . Kernspintomographie · Aortensteifigkeit . Distensibilität

\section{Aortic imaging as key to therapy for genetic aortic syndromes}

\section{Abstract}

Background. Imaging is a key factor in the management of individuals with genetic aortic diseases, such as Marfan syndrome, vascular Ehlers-Danlos syndrome and LoeysDietz syndrome. Imaging is essential for identifying individuals with genetic aortic syndromes and for diagnostics of acute aortic syndromes in affected individuals. Imaging modalities for elective and emergency diagnostics comprise transthoracic echocardiography, transesophageal echocardiography, computed tomography (CT) with $\mathrm{CT}$ angiography and magnetic resonance imaging (MRI) with MR angiography. Objectives. The importance of echocardiographic M-mode image segmentation method for measurement of changes in stiffness of the aorta.

Materials und Methods. Study on measurement of stiffness of the aorta in Marfan syndrome in comparison to healthy subjects.
Results. Using the non-invasive semiautomatic echocardiographic M-mode image segmentation method, reduction in the elastic properties of the aorta can be exactly and objectively measured in young patients with Marfan syndrome.

Conclusions. Imaging procedures are essential for identifying genetic aortic diseases, for stratifying aortic risk and for diagnosing acute aortic syndrome. For reasons of radiation protection, echocardiography and MRI should be preferentially used for follow-up in young patients.

Keywords

Marfan syndrome - Echocardiography . Magnetic resonance imaging $\cdot$ Aortic stiffness · Distensibility 


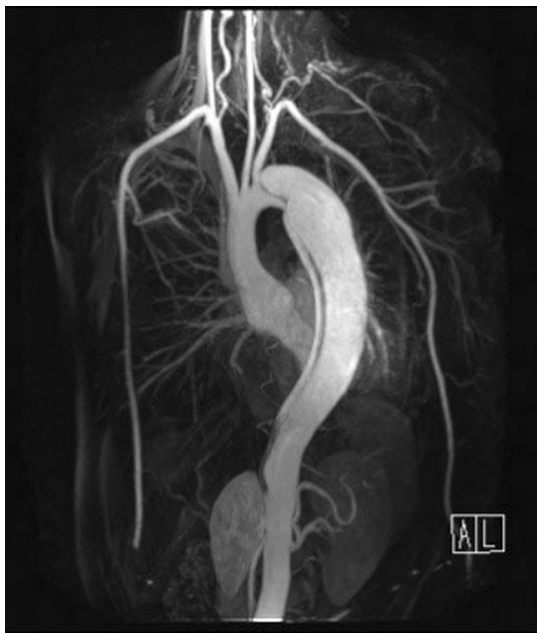

Abb. 1 A Patientin mit Stanford-Typ-B-Dissektion

Normwerte bezogen werden. Besonders für Untersuchungen von Kindern im Wachstum ist das essenziell. Viel diskutiert wurde der Gebrauch von Z-Scores. Diese sind für Studien von Nutzen. Intraindividuelle Veränderungen von Z-Scores sind bei Körpergewichtsschwankungen vor allem bei Erwachsenen oft schwierig zu interpretieren, weshalb Z-Scores niemals isoliert ohne absolute Aortenmesswertdifferenzen betrachtet werden sollen. Hilfen für die Kalkulation der Z-Scores finden sich im Internet unter http://parameterz. blogspot.com. Bei der Verwendung von Normwertnomogrammen und Normwerttabellen ist darauf $\mathrm{zu}$ achten, dass die Akquisition und Messung der Aorta mit derselben Messmethode erfolgt wie in der den Normwerten zugrundeliegenden Untersuchung. In der Tat existieren verschiedene Normwerte, je nach Verwendung der Leading-edge-Methode, bei der von der vorderen Vorderwand- zur vorderen Hinterwandkontur der Aorta gemessen wird, um den Fehler, der durch mehr oder weniger Ultraschall-Gain entsteht, zu eliminieren, oder der Messung der Aorteninnendurchmesser oder der Messung der Aortenaußendurchmesser.

\section{Steifheitsmessungen der Aorta}

In der chirurgischen Literatur wird die Indikation zum operativen Aortenersatz

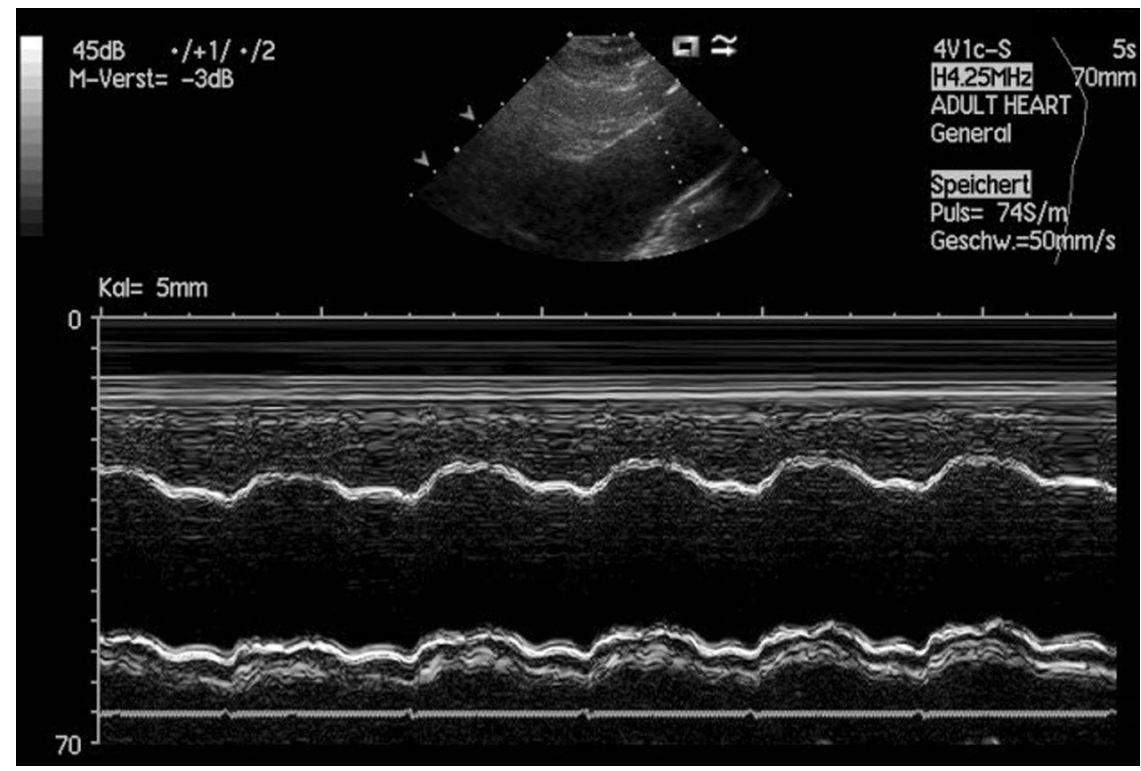

Abb. 2 A M-Mode der Aorta ascendens

fast ausschließlich von absoluten Aortendurchmessern abhängig gemacht. Diese Grenzen basieren auf Erfahrungswerten und der Tatsache, dass der mechanische Aortenwandstress und damit die Gefahr für eine Aortendissektion- und -ruptur entsprechend dem LaplaceGesetz mit dem Quadrat des Aortendurchmessers steigt. Unwahrscheinlich ist, dass der Aortenwandstress einer kleingewachsenen Asiatin bei gleichem Aortendurchmesser dem eines großgewachsenen Nordeuropäers entspricht. Deshalb werden diese absoluten Durchmessergrenzen auf die Körperoberfläche bezogen, was insbesondere bei Frauen wichtig ist, um hier eine systematische Verzögerung des Operationszeitpunkts $\mathrm{zu}$ vermeiden.

Wichtiger noch ist die Tatsache, dass die Stabilität des Gefäßes bei Dilatation nicht nur vom Durchmesser, sondern auch von der Wanddicke und Wandstabilität der Aorta abhängen. Diese Einflussgrößen bleiben bei rein anatomischen Vermessung der Aorta systematisch unberücksichtigt.

Einen Ausweg aus diesem Dilemma bieten funktionelle Untersuchungen der Aorta. Diese sind mittels M-Mode-Echokardiographie oder Cine-MR möglich.

Stefanadis et al. [6] bestimmten 1987 erstmals mittels Herzkatheterisierung und Angiographie der Aorta aus Druckund Durchmesserwerten die Dehnbar- keit (Distensibilität) der Aorta. Eine methodische Verbesserung gelang durch eine spezielle Kathetertechnik, mit der Durchmesser und Druck am selben Ort der Aorta zeitgleich bestimmt werden konnten [7]. Kawasaki et al. [8] gelang die nichtinvasive Bestimmung der Steifheit verschiedener Arterien mittels ultrasonographischer Durchmesserbestimmung in diversen Arterien und Manschettenblutdruckmessung in der Arteria radialis. Stefanadis et al. [9] fanden eine gute Korrelation von invasiven und nichtinvasiven (M-Mode echokardiographischen) Distensibilitätsmessungen in der Aorta ascendens.

Bei Erwachsenen mit Marfan-Syndrom stellten verschiedene Autoren mit nichtinvasiven Verfahren (Echokardiographie oder Magnetresonanztomographie) verminderte elastische Eigenschaften der Aorta fest [10-12]. Bei Kindern und Jugendlichen zeigten sich ebenfalls verminderte Elastizitätswerte der Aorta [13-15]. Diese nichtinvasiven Untersuchungen beruhten allerdings auf manuellen Messungen der enddiastolischen (minimalen) und endsystolischen (maximalen) Aortendurchmesser, wodurch die Ergebnisse untersucherabhängig sind.

Exakter reproduzierbar sind Untersuchungen, in denen die Aortendurchmesserbestimmung kontinuierlich mit einer Konturfindungssoftware mit hoher zeitlicher und örtlicher Auflösung aus 


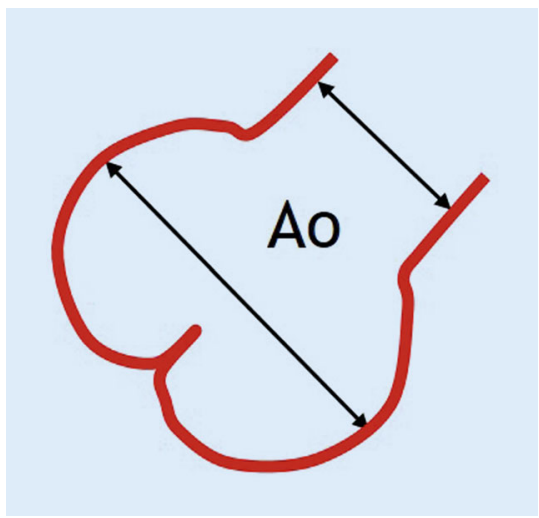

Abb. $3 \Delta$ Hoch parasternaler Längsschnitt durch die Aortenwurzel. Schnittebene für M-Mode-echokardiographische Durchmesserbestimmung in der Aorta ascendens (nach [17])

M-Mode-echokardiographischen Bildern erfolgt ([16]; - Abb. 2). Die genaue Schnittführung in der Aorta ascendens zeigt • Abb. 3.

Zur Berechnung der Distensibilität [13] und des Steifheitsindex [10] wurden die folgenden Formeln angegeben, wobei $A_{s}$ der systolischen und $A_{d}$ der enddiastolischen Aortenquerschnittsfläche, $D_{s}$ und $D_{d}$ dem systolischen und dem enddiastoischen Aortendurchmesser, sowie $P_{s}$ und $P_{d}$ dem systolischen und dem diastolischen Blutdruck, gemessen in $\mathrm{mm} \mathrm{Hg}$, entsprechen, und die Querschnittsfläche nach der Formel $(\mathrm{D} / 2)^{2} \pi$ kalkuliert werden kann:

Distensibilität $=$

$$
\begin{aligned}
& \frac{A_{s}-A_{d}}{A_{d} \cdot\left(P_{s}-P_{d}\right) \cdot 1333} \cdot 10^{7} \\
& {\left[\mathrm{kPa}^{-1} \cdot 10^{-3}\right]}
\end{aligned}
$$

Steifheitsindex $=\frac{\operatorname{In}\left(P_{s} / P_{d}\right)}{\left(D_{s}-D_{d}\right) / D_{d}}[]$

Zur diagnostischen Aussagekraft der Aortenelastizitätsparameter gibt es kaum Daten. Bekannt ist, dass die elastischen Eigenschaften der Aorta bei Gesunden mit dem Alter abnehmen [18], während kardiale Ereignisse im Alter zunehmen. Außerdem ist die Aortenelastizität durch Pharmaka wie $\beta$-Blocker und Angiotensin-II-Typ-1-Rezeptorblocker (Sartane) beeinflussbar [19]. Die elastischen Eigenschaften stellen daher einen Prognostikator für kardiale Ereignisse

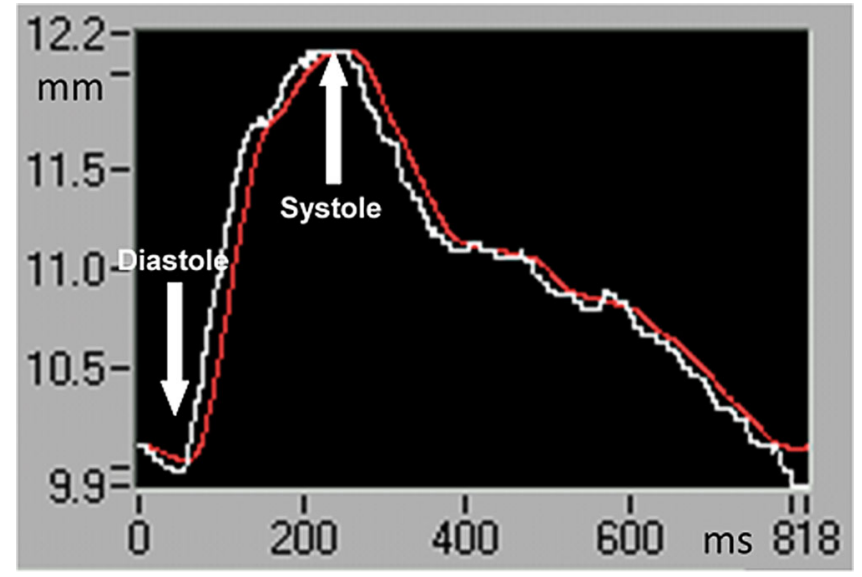

Abb. $4<$ Gemittelte DurchmesserZeit-Kurve der Aorta ascendens dar und sind für das Therapiemonitoring einsetzbar [19].

\section{Beispiel einer Studie zur aortalen Steifheitsmessung}

Wir stellen hier exemplarisch eine Studie zur Steifheitsmessung der Aorta bei Marfan-Syndrom im Vergleich zu gesunden Probanden dar. In dieser prospektiven, kontrollierten Studie wurden 19 Patienten mit Marfan-Syndrom entsprechend der Genter Nosologie im Alter von 17,7 \pm 9,5 Jahren vor Beginn einer Therapie mit $\beta$-Blockern sowie 19 gleichaltrige herzgesunde Kontrollpersonen untersucht [16]. Eine Mutationsanalyse des FBN1Genes bestätigte die Diagnose bei 18 der 19 Patienten, nur in einem Fall fand sich keine Mutation. M-Mode-echokardiographisch wurden die Durchmesser des Bulbus aortae, der Aorta ascendens 1-2 cm distal des sinutubulären Übergangs und der Aorta descendens proximal des Abgangs des Truncus coeliacus nach der Leading-edge-Methode über mindestens 5 Herzzyklen registriert. Mittels einer eigens entwickelten Autokonturfindungssoftware bestimmten wir die Aortendurchmesser kontinuierlich mit einer zeitlichen Auflösung von 6 ms pro Pixel und einer örtlichen Auflösung von $0,2 \mathrm{~mm}$ pro Pixel (• Abb. 4). Der Blutdruck wurde oszillometrisch am rechten Arm dreimal unmittelbar vor der M-Mode-Registrierung bestimmt. Neben absoluten und auf die Körper- oberfläche bezogenen, enddiastolischen und systolischen Aortendurchmessern wurden aus den AortendurchmesserZeit-Kurven und Blutdruckwerten in der Aorta aszendens und in der Aorta deszendens die elastischen Parameter Distensibilität, Steifheitsindex und maximale systolische Querschnittsflächenzunahme (MSAI = maximaler Anstieg der Querschnittsflächen-Zeit-Kurve) bestimmt.

Bei den Patienten mit Marfan-Syndrom zeigte sich in der Aorta ascendens eine Verminderung der elastischen Eigenschaften auf etwa die Hälfte des Normalen, in der Aorta descendens ebenfalls eine signifikante Elastizitätsminderung um etwa ein Drittel. Distensibilität und MSAI korrelierten eng miteinander.

Mittels der Vektoraortographie konnte graphisch dargestellt werden, in welchem untersuchten Aortenabschnitt der Patienten die herzzyklusabhängigen Durchmesseränderungen stärker reduziert sind. Drei von 19 Patienten (16\%) zeigten eine vorwiegende Elastizitätsverminderung in der Aorta descendens, bei den restlichen 16 (84\%) war die Aorta ascendens stärker betroffen.

Die diagnostische Aussagekraft der kardiovaskulären Parameter wurde mittels einer logistischen Regressionsanalyse untersucht. Das aussagekräftigste multipel binär logistische Regressionsmodell schloss ausschließlich aortale Parameter ein (auf die Körperoberfläche bezogene Durchmesser des Bulbus aortae und der 
Aorta ascendens sowie Distensibilität der Aorta ascendens) und unterschied mit einer Sensitivität von $95 \%$ sowie einer Spezifität von $100 \%$ zwischen Patienten mit Marfan-Syndrom und Kontrollpersonen.

\section{Klinische Bedeutung von aortalen Steifheitsmessungen}

Die Daten dieser Studie dokumentieren beispielhaft die diagnostische Aussagekraft von aortalen Steifheitsmessungen. Mit unserer nichtinvasiven, semiautomatisierten M-Mode echokardiographischen Bildsegmentationsmethode konnten wir die Verminderung der elastischen Eigenschaften der Aorta bei jungen Patienten mit Marfan-Syndrom genau und objektiv erfassen. Dies ermöglicht verlässliche Verlaufsuntersuchungen, die wesentlich sind, da die Zunahme der Aortendurchmesser eine prognostische Bedeutung hat [20]. Die Minderheit der Patienten mit vorwiegenden Veränderungen in der Aorta descendens könnte dem Anteil der Patienten entsprechen, bei dem ein Risiko für Aneurysmen und Dissektionen der Aorta descendens besteht [4].

Da sich Einschränkungen der Aortenelastizität auch bei Patienten mit grenzwertigen und normalen Aortendurchmessern fanden, stellt das logistische Regressionsmodell, dessen Anwendung wesentlich einfacher ist als die Durchführung der kompletten klinischen oder molekulargenetischen Diagnostik, einen sensiblen und spezifischen Marker für das Vorhandensein eines Marfan-Syndroms dar. Beim Verdacht auf Vorliegen eines Marfan-Syndroms kann es helfen, über die Notwendigkeit weiterer Verlaufsuntersuchungen $\mathrm{zu}$ entscheiden. Zudem kann dieses Modell in Verlaufsuntersuchungen den Erfolg einer medikamentösen Therapie bzw. die Progredienz der Aortenveränderungen zeigen und somit auch im Hinblick auf die Notwendigkeit eines prophylaktischen Aortenersatzes als Entscheidungshilfe eingesetzt werden.

\section{Differenzialdiagnosen des Marfan-Syndroms}

Bei milderen Fibrillinopathien wie dem Mitralklappenprolaps-Syndrom findet sich keine Aortendilatation, beim MASSPhänotyp kann eine milde Aortendilatation vorhanden sein. Eine Progredienz ist nicht gegeben. Die Aortenelastizitätsveränderungen sind milde.

Das Loeys-Dietz-Syndrom ist in seinen aortalen Komplikationen aggressiver und unberechenbarer, da schon in jungem Alter und bei geringer Aortendilatation Rupturen der Aorta und anderer Arterien vorkommen. Aortendissektionen innerhalb des ersten Lebensjahres oder intrazerebrale Blutungen im Kleinkindesalter treten auf [21].

Bei den sehr seltenen erblichen Aortendilatationssyndromen treten extraaortale Manifestationen in den Hintergrund. Sie werden durch folgende Gene verursacht: ACTA2, Dissektionen in der proximalen und distalen Aorta kommen vor; MYH11, assoziiert mit Ductus arteriosus; FBLN4, assoziiert mit Cutis laxa; GLUT10, das Arterielle Tortuositäts-Syndrom mit sehrvariablem Verlauf und Tortuosität auch kleinerer Arterien bereits nach der Geburt oder erst im höheren Alter.

Bei den meisten Formen des EhlersDanlos-Syndroms sind wenig Aortenveränderungen festzustellen. Ausnahmen sind vaskuläre Verlaufsformen (Typ 4 und Typ 7b). Es finden sich Komplikationen im Aortenbogen und in der deszendierenden Aorta sowie in den mittelgroßen Arterien, die normalkalibrig sind. Bei Eingriffen ist mit schwer stillbaren Blutungen zu rechnen.

Patientinnen mit Turner-Syndrom erhalten oft Wachstumshormon- und Östrogenpräparate, die zu einer Progredienz der Aortendilatation führen können. Hormonelle Stimulationen im Rahmen des Oozytentransfers sind wegen des deutlich erhöhten Aortenrupturrisikos kritisch zu hinterfragen. Bikuspide Aortenklappe, Aortenisthmusstenose und arterielle Hypertonie sind Risikofaktoren für aortale Komplikationen.

\section{Allgemeine Prinzipien der Therapie}

Für alle Schwangerschaften mit Aortopathie ist eine kardiologische Mitbetreuung mit engmaschiger Bildgebung der Aorta und exakter Blutdruckeinstellung essenziell. Dabei ist ein niedrig normaler Blutdruck anzustreben. Ein mütterlicher Aortendurchmesser von über $40 \mathrm{~mm}$ zum Zeitpunkt der Geburt gilt als Indikationskriterium für eine Sectio caesarea. Diese sollte idealerweise in einem Zentrum mit Herzchirurgie durchgeführt werden.

Die medikamentöse Therapie der Aortendilatation mit $\beta$-Blockern und Angiotensin-II-Typ-1-Rezeptor-Blockern hat zum Ziel, den Stress, den jeder Herzschlag auf die Aortenwand ausübt, zu vermindern. Eine Verminderung des Blutdrucks sowie ein langsamerer Anstieg des systolischen Blutdrucks können das Fortschreiten der Aortendilatation bremsen. Verlaufsuntersuchungen haben gezeigt, dass ein früher Therapiebeginn im Kindesalter bei milder Aortendilatation effizienter ist als ein späterer Beginn bei bereits deutlicher Aortendilatation [22]. So kann die Notwendigkeit prophylaktischer Aortenersatzoperationen um Jahre verzögert werden. Die Therapie mit $\beta$-Blockern ist insbesondere bei Kindern effektiv. Bei fortgeschrittener Aortendilatation ist eine Kombination von $\beta$-Blockern und Sartanen möglich. Auch nach Aortenersatzoperationen sollten $\beta$-Blocker oder Sartane Teil der Therapie sein.

Erforderlich für die frühe Diagnose ist, möglicherweise betroffene Verwandte von Patienten mit erblicher Aortopathie einer frühzeitigen echokardiographischen Screeninguntersuchung zuzuführen.

Die molekulargenetischen Untersuchung der Patienten aus EDTA-Blut ermöglicht, bei Verwandten die bekannte Mutation gezielt zu suchen.

Lifestyle-Modifikationen bestehen darin, das Heben schwerer Gewichte, Stöße gegen den Thorax und maximale körperliche Anstrengungen zu meiden. 


\section{Fazit für die Praxis}

- Dysproportionierter Hochwuchs, Arachnodaktylie, Kielbrust, Skoliose oder Ektopia lentis lassen an eine erbliche Bindegewebeschwäche denken. Eine symptomfreie Aortendilatation soll frühestmöglich erkannt und behandelt werden, da sie schon bei jungen Patienten zu einer lebensbedrohlichen Aortendissektion und -ruptur führen kann.

- Die transthorakale Echokardiographie dient als Screeningtool für die Aortendilatation. Die Messwerte werden auf die Körperoberfläche bezogen.

- Die medikamentöse Therapie besteht in der Gabe von $\beta$-Blockern (z. B. Atenolol, Metoprolol) und/oder Angiotensin-II-Typ-1-Rezeptorblockern (Sartanen: z. B. Candesartan, Losartan) und kann eine prophylaktische Aortenwurzelersatzoperation um Jahre hinauszögern.

- Transösophageale Echokardiographie, CT mit CT-Angiographie und MRT mit MR-Angiographie erlauben die Diagnostik einer Aortendissektion, die bei anhaltenden Thoraxschmerzen rasch abzuklären und zu behandeln ist. Vor einer elektiven Aortenwurzelersatzoperation ist eine Schnittbilduntersuchung der gesamten Aorta durchzuführen.

- Aus Strahlenschutzgründen sollen für Verlaufsuntersuchungen junger Patienten bevorzugt Echokardiographie und MRT eingesetzt werden.

- Da eine Aortendilatation während einer Schwangerschaft rasch zunehmen kann, sind regelmäßige Untersuchungen der Aorta und eine exakte Blutdruckeinstellung empfohlen.

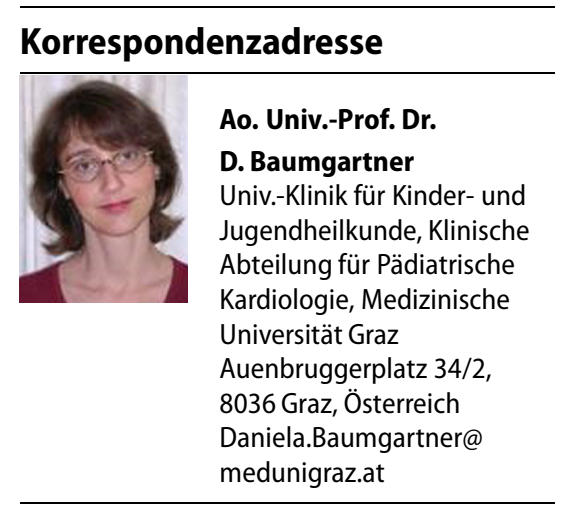

Open access funding provided by Medical University of Graz.

\section{Einhaltung ethischer Richtlinien}

Interessenkonflikt. D. Baumgartner und Y. von Kodolitsch geben an, dass kein Interessenkonflikt besteht.

Dieser Beitrag beinhaltet keine von den Autoren durchgeführten Studien an Menschen oder Tieren.

Open Access. Dieser Artikel wird unter der Creative Commons Namensnennung 4.0 International Lizenz (http://creativecommons.org/licenses/by/4.0/deed. de) veröffentlicht, welche die Nutzung, Vervielfältigung, Bearbeitung, Verbreitung und Wiedergabe in jeglichem Medium und Format erlaubt, sofern Sie den/die ursprünglichen Autor(en) und die Quelle ordnungsgemäßnennen, einen Link zur Creative Commons Lizenz beifügen und angeben, ob Änderungen vorgenommen wurden.

\section{Literatur}

1. Roman MJ, Devereux RB, Kramer-Fox R, O'Loughlin J (1989) Two-dimensional echocardiographic aortic root dimensions in normal children and adults. Am J Cardiol 64:507-512

2. Spittell PC, Spittell JA Jr, Joyce JW et al (1993) Clinical features and differential diagnosis of aortic dissection: experience with 236 cases (1980 through 1990). Mayo Clin Proc 68:642-651

3. Debakey ME, Henly WS, Cooley DA et al (1965) Surgical management of dissecting aneurysms of the aorta. J Thorac Cardiovasc Surg 49:130-149

4. Gott VL, Cameron DE, Alejo DE et al (2002) Aortic root replacement in 271 Marfan patients: A 24-year experience. Ann Thorac Surg 73:438-443

5. Gott VL, Greene PS, Alejo DE et al (1999) Replacement of the aortic root in patients with Marfan's syndrome. NEngl J Med 340:1307-1313

6. Stefanadis C, Wooley CF, Bush CA, Kolibash AJ, Boudoulas H (1987) Aortic distensibility abnormalities in coronary artery disease. Am J Cardiol 59:1300-1304

7. Stefanadis C, Stratos C, Vlachopoulos C et al (1995) Pressure-diameter relation of the human aorta. A new method of determination by the application of a special ultrasonic dimension catheter. Circulation 92:2210-2219

8. Kawasaki T, Sasayama S, Yagi SI, Asakawa T, Hirai T (1987) Non-invasive assessment of the age related changes in stiffness of major branches of the human arteries. Cardiovasc Res 21:678-687

9. Stefanadis C, Stratos C, Boudoulas H, Kourouklis C, Toutouzas P (1990) Distensibility of the ascending aorta: Comparison of invasive and non-invasive techniques in healthy men and in men with coronary artery disease. Eur Heart J 11:990-996

10. Hirata K, Triposkiadis F, Sparks E, Bowen J, Wooley C, Boudoulas H (1991) The Marfan syndrome: Abnormal aortic elastic properties. J Am Coll Cardiol 18:57-63

11. Adams JN, Brooks M, Redpath TW et al (1995) Aortic distensibility and stiffness index measured by magnetic resonance imaging in patients with Marfan's syndrome. Br Heart J73:265-269

12. Nollen GJ, Groenink M, Tijssen JGP et al (2004) Aortic stiffness and diameter predict progressive aortic dilatation in patients with Marfan syndrome. Eur Heart J25:1146-1152

13. Savolainen A, Keto $P$, Hekali $P$ et al (1992) Aortic distensibility in children with the Marfan syndrome. Am J Cardiol 70:691-693

14. Reed CM, Fox ME, Alpert BS (1993) Aortic biomechanical properties in pediatric patients with the Marfan syndrome, and the effects of atenolol. Am J Cardiol 71:606-608

15. Jeremy RW, Huang $\mathrm{H}$, Hwa J, McCarron $\mathrm{H}$, Hughes CF, Richards JG (1994) Relation between age, arterial distensibility, and aortic dilatation in the Marfan syndrome. Am J Cardiol 74:369-373

16. Baumgartner $D$, Baumgartner $C$, Mátyás $G$ et al (2005) Diagnostic power of aortic elastic properties in young patients with Marfan syndrome. J Thorac Cardiovasc Surg 129:730-739

17. Vogt M, Kühn A, Baumgartner D et al (2005) Impaired elastic properties of the ascending aorta in newborns before and early after successful coarctation repair. Proof of a systemic vascular disease of the prestenotic arteries? Circulation 111:3269-3273

18. Avolio AP, Chen SG, Wang RP, Zhang CL, Li MF, O'Rourke MF (1983) Effects of aging on changing arterial compliance and left ventricular load in a northern Chinese urban community. Circulation 68:50-58

19. Vlachopoulos C, Aznaouridis K, Stefanadis C (2006) Clinical appraisal of arterial stiffness: the Argonauts in front of the Golden Fleece. Heart 92:1544-1550. (Published Online First: 9 December 2005) doi:10. 1136/hrt.2005.067025

20. Groenink M, Rozendaal L, Naeff MSJ et al (1998) Marfan syndrome in children and adolescents: Predictive and prognostic value of aortic root growth for screening for aortic complications. Heart 80:163-169

21. Williams JA, Loeys BL, Nwakanma LU, Dietz HC, Spevak PJ, Patel ND, Francois K, DeBacker J, Gott VL, Vricella LA, Cameron DE (2007) Early surgical experience with Loeys-Dietz: A new syndrome of aggressive thoracic aortic aneurysm disease. Ann Thorac Surg 83:757-763

22. Baumgartner D, Baumgartner C, Schermer E, Engl G, Schweigmann U, Matyas G et al (2006) Different patterns of aortic wall elasticity in patients with Marfan syndrome: A noninvasive follos-up study. JThorac Cardiovasc Surg 132:811-819 